\title{
Comparison between taans cerebellar diameter, biparietal diameter and femur length for gestational age measurement accuracy in the $3^{\text {rd }}$ trimester
}

\author{
Alsaied Abdelmaksod Askr, Mahmoud Salah Mahmoud, Asmaa Ahmed Mahmoud Salloum* \\ Department of Obstetrics and Gynecology, Faculty of Medicine, Al-Azhar University \\ *Corresponding author: Asmaa Ahmed Mahmoud Salloum, Mobile: 00201005854475, E-Mail: drasmaa822@gmail.com
}

\begin{abstract}
:
Background: the provision of obstetric and neonatal care, as well as the public health monitoring of pregnancy outcomes, relies upon the accurate determination of gestational age. Uncertain gestational age has been associated with adverse pregnancy outcomes including low birth weight, preterm delivery and perinatal mortality and independent of maternal characteristics. Many patients in Egypt due to socio-economic reasons come for their first antenatal visit in the third trimester. Most of them are uneducated and come from remote areas. Also, many being lactating mothers are unsure of their LMP or have irregular cycles. Because of non-availability of any dating scans or earlier ultrasound and uncertainty in LMP, it becomes very difficult to calculate their due dates, so many pregnancies considered to be preterm or posterm were wrongly classified. The use of ultrasonography has significantly improved evaluation of fetal growth and development and had permitted prenatal diagnosis of a variety of congenital malformations. Ultrasonographic fetal biometry is highly reliable in the first and second trimester of pregnancy, but reliability of any ultrasound method greatly diminishes as gestational age advances, in the third trimester, reliability of any single ultrasound parameter alone is poor without correlation with other parameters. Objective: in our study, TCD was compared to FL and BPD in accuracy of assessment of gestational age in the third trimester of pregnancy. We correlated between the three parameters, Transcerebellar (TCD) and Biparietal diameter (BPD) and Femur length (FL), for accurate determination of gestational age in third trimester of pregnancy. Methods: a total of 200 pregnant women in the third trimester pregnancy were assessed in this observational study in Department of Obstetrics and Gynecology, Damietta Hospital, Al-Azhar University (Outpatient) by two-dimensional ultrasound to assess the accurate method for assessment of gestational age in the third trimester of pregnancy. Examinations were performed after a verbal consent from the patient with the patient lying in the dorsal supine position. Two dimensional ultrasounds were carried out. Fetal biometry and amniotic fluid volume were assessed. All women included in this study were subjected to history taking and clinical examination. Results: we found that out of 200 patients, TCD gave correct assessment of gestational age within 3 days in 118 patients (59\%) and within 1 week in 180 patients (90\%). While, FL gave correct assessment of gestational age within 3 days in 92 patients (46\%) and within 1 week in 160 patients $(80 \%)$. The least accurate was BPD that gave correct assessment of gestational age within 3 days in 59 patients $(29.5 \%)$ and within 1 week in 120 patients $(60 \%)$.

Conclusion: from this study, we concluded that TCD was the most accurate method for assessment of gestational age in the third trimester followed by FL and the least accurate was the BPD. Also, by combining accuracy of TCD (90\%) and that of FL (80\%) we can be near certain of gestational age in most of our patients even if they are unsure of their dates.
\end{abstract}

Recommendations: measuring TCD as routine in the third trimester as it has the same accuracy as the current fetal biometry (BPD and FL).

Keywords: TCD, LMP, BPD, FL, GA

\section{Introduction:}

The pregnancy date estimation is mandatory for the pregnant ladies in order to have the expect time of delivery in which various tests will be taken to achieve the estimated time. There are methods that used to determine the gestational age including menstrual history, clinical examination and the ultra sonography ${ }^{(\mathbf{1})}$. Accurate determination of the gestational dating is considered to be one of the most beneficial assessments in pregnancy, which is dependent on determination whether the patient in gestation or not .In order to decrease the biologic variability among fetuses traditional biometry, ancillary biometric also non biometric measurements will be used in addition those measurements can also be used for the assessment of the gestational age and maturity of the fetal lung and some specific 
clinical situations as cases of oligohydramnios that manifested by fetal head and abdominal compression resulting in difficult determination of accurate abdominal circumference and biparietal diameter ${ }^{(2)}$.

Aim of the work:

This study aimed to assess the accuracy of transcerebellar diameter (T.C.D.) measurement in estimation of the gestational age during the third trimester compared to the current fetal biometric measurements (Femur length and biparietal diameter) according to last menstrual period.

Patients and methods:

This observational study was conducted on 200 pregnant women at Department of Obstetrics and Gynecology, Damietta Hospital, Al-Azhar University (Outpatient), all patients were in the third trimester of pregnancy. The study was approved by the Ethics Board of Al-Azhar University.

The inclusion criteria were:

- Women in childbearing period

- Singleton

- Uncomplicated pregnancy

- At 29-36 weeks of pregnancy calculated by the first day of last menstrual periods.

\section{The Exclusion criteria were:}

- Patients who were unsure of dates

- Those with fetus with congenital anomalies

- Intrauterine fetal death

- Patients with multiple gestation

- Patients with medical disorders like hypertension and diabetes were excluded.

\section{Method:}

Patients included in this study were subjected to verbal consent obtained from the pregnant women who were included in the study. Full history was taking and included the name, age, occupation and address. Obstetric history and $1^{\text {st }}$ day of last menstrual period (LMP), gestational age documentation, medical or operative history, and any drug allergy or obstetric or operative complication were verified.Regarding the ultrasound it was done at clinic of Damietta Hospital, Al-Azhar university, mainly to measure the fetal transcerebellar diameter, biparietal diameter and femur length as a parameter of gestational age estimation. The technique of ultrasound conducted was included to perform a transabdominal ultrasound on all patients, while women were in a tilted position with the head of the bed raised 30 degrees and with a small pillow under the right loin. Measurement of the biparietal diameter was taken in the lateral ventricles view, a rugby-football-shaped skull, rounded at the back (occiput) and more pointed at the front (synciput) with along midline equidistant from the proximal and distal scale echoes. The cavum septum pellucidum bisected the midline onelthird of the distance from the synciput to the occiput. The two anterior horns of the lateral ventricles symmetrically placed about the midline. All or part of the posterior horns of the lateral ventricles symmetrically placed about the midline. The BPD included the thickness of only the upper parietal bone (outer to outer measurement).Regarding measurement of the transcerebellar diameter, obtaining the transthalamic view of BPD then rotation of the probe slightly downwards, toward the fetal neck and the posterior horns of the lateral ventricles would be disappeared from the view to be replaced by the cerebellum. TCD measured at 90 degree to the long axis of the cerebellum across its widest point, by the use of the outer to outer method. Regarding measurement of the femur length, FDL was imaged optimally with both ends of the ossified metaphysis clearly visible. The longest axis of the ossified diaphysis is measured. The same technique as that used to establish the reference chart should be used with regard to the angle between the femur and the insonating ultrasound beams. An angle of insonation between $45^{\circ}$ and $90^{\circ}$ is typical. Regarding the Caliper placement, each caliper is placed at the ends ofthe ossified diaphysis without including the distal femoralepiphysis if it is visible. This measurementshould exclude triangular spur artifacts that can falselyextend the diaphysis length.

Ultrasound device: voluson 730 ultrasound machine, Astria software.

\section{Statistical methods:}

Data were statistically described in terms of mean \pm standard deviation ( \pm SD), and range or frequencies (number of cases) and percentages when appropriate. Comparison between the different methods of estimating gestational age was done using paired t- test. Accuracy of different estimation parameters in relation to the LMP parameter was done within 1 week error. $p$-values less than 0.05 was considered statistically significant. All statistical calculations were done by using computer program SPSS (Statistical Package 
for the Social Science; SPSS Inc., Chicago, IL, USA) release 15 for Microsoft Windows (2006).

Results:

The majority of the patients in our study were belonged to the age group 20 to 28 years $(65 \%)$ with minimum 17 years and maximum 37 years. In our study, also 55 patients out of 200 were primigravida and 145 patients were
multigravida.Our results showed that the percentages of accurate assessment of gestational age within 3 days were as follow: out of 200 patients TCD gave accurate assessment within 3 days in 118 patients (59\%).FL gave accurate assessment within 3 days in 92 patients $(46 \%)$ while BPD gave accurate assessment within 3 days in 59 patients (29.5\%) (Table 1).

Table 1- Percentages of correct as assessment within 3 days

\begin{tabular}{|c|c|c|c|}
\hline Type of measurement & $\begin{array}{c}\text { Number of patients } \\
\text { positive }\end{array}$ & $\begin{array}{c}\text { Number of patients } \\
\text { negative }\end{array}$ & Positive percentage \\
\hline TCD & 118 & 82 & $59 \%$ \\
\hline BPD & 59 & 141 & $29.5 \%$ \\
\hline FL & 92 & 108 & $46 \%$ \\
\hline
\end{tabular}

Chi square test was performed to determine the $\mathrm{P}$ value and comparing between the 3 results. Comparison between the percentages of correct assessment within 3 days by TCD and BPD were as follow (Table 2):

Table 2-P value comparing TCD with BPD within 3 days

\begin{tabular}{|c|c|c|c|c|c|c|}
\hline \multirow{2}{*}{} & \multicolumn{3}{|c|}{ BPD } & \multicolumn{2}{c|}{ TCD } & \multirow{2}{*}{ P value } \\
\cline { 3 - 7 } & & Count & \% & Count & \% & \multirow{2}{*}{$<0.001$} \\
\hline \multirow{2}{*}{ Accuracy } & yes & 59 & $29.5 \%$ & 118 & $59.0 \%$ & \\
\cline { 2 - 6 } & no & 141 & $70.5 \%$ & 82 & $41.0 \%$ & \\
\hline
\end{tabular}

The $\mathrm{P}$ value was found to be $\mathbf{< 0 . 0 0 1}$ showing that there was highly significant difference between correct assessment within 3 days by BPD and by TCD.

Comparison between the percentages of correct assessment within 3 days by the TCD and FL was detected in table 3.

Table 3- P value comparing TCD with FL within 3 days

\begin{tabular}{|c|c|c|c|c|c|c|}
\hline \multirow{2}{*}{} & \multicolumn{3}{|c|}{ TCD } & \multicolumn{2}{c|}{ FL } & \multirow{2}{*}{ P value } \\
\cline { 2 - 7 } & & Count & \% & Count & \% & \multirow{2}{*}{0.009} \\
\hline \multirow{3}{*}{ Accuracy } & yes & 118 & $59.0 \%$ & 92 & $46.0 \%$ & $54.0 \%$ \\
\cline { 2 - 7 } & no & 82 & $41.0 \%$ & 108 & & \\
\hline
\end{tabular}

The $\mathrm{P}$ value was found to be 0.009 and showed that there was a significant difference between the correct assessment within 3 days by FL and TCD.

Comparison between the percentages of correct assessment within 3 days by the BPD and FL was shown in table 4.

Table 4- $P$ value comparing FLwith BPD within 3 days

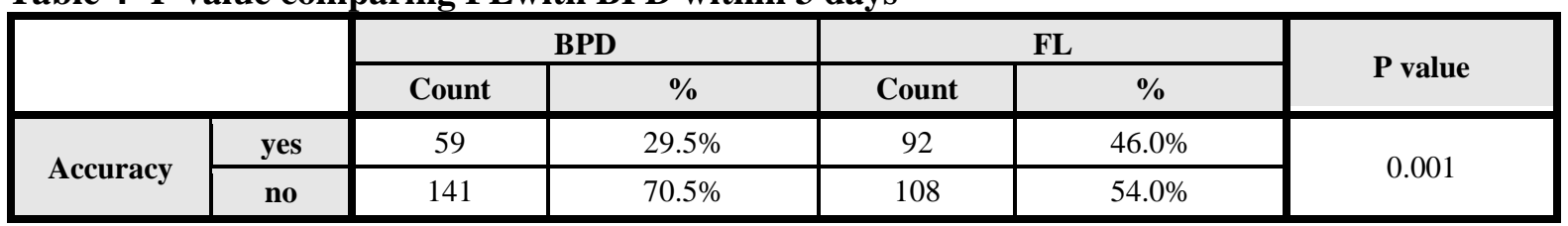

The $\mathrm{P}$ value was found to be 0.001 and showed that there was a shighly significant difference between correct assessment within 3 days by BPD and by FL.

While, upon calculating the percentage of accurate assessment of gestational age by the 3 parameters (TCD, BPD and FL) within one week the following results were obtained .Out of 200 patients, TCD gave correct assessment of gestational age in 180 patients $(90 \%)$. While FL gave correct assessment of gestational age in 160 patients $(80 \%)$. Finally BPD gave correct assessment of gestational age in 120 patients $(60 \%)$ (Table 5). 
Table 5- Percentages of correct as assessment within one week

\begin{tabular}{|c|c|c|c|}
\hline Type of measurement & Number of patients positive & Number of patients negative & Positive percentage \\
\hline TCD & 180 & 20 & $90 \%$ \\
\hline BPD & 120 & 80 & $60 \%$ \\
\hline FL & 160 & 40 & $80 \%$ \\
\hline
\end{tabular}

Comparison between the percentages of correct assessment within 1 week by the TCD and BPD was recorded in table 6.

Table 6- $P$ value comparing TCD with BPD within 1 week

\begin{tabular}{|c|c|c|c|c|c|c|}
\hline \multirow{2}{*}{} & \multicolumn{2}{|c|}{ BPD } & \multicolumn{2}{c|}{ TCD } & \multirow{2}{*}{ P value } \\
\cline { 3 - 7 } & & Count & \% & Count & $\%$ & \\
\hline \multirow{2}{*}{ Accuracy } & Yes & 120 & $60.0 \%$ & 180 & $90 \%$ & \multirow{2}{*}{$<0.001$} \\
\cline { 2 - 6 } & No & 80 & $40.0 \%$ & 20 & $10.0 \%$ & \\
\hline
\end{tabular}

The $\mathrm{P}$ value was found to be $<0.001$ and showed that there was a highly significant difference between the correct assessment within 1 week by BPD and by TCD.

Comparison between the percentage of correct assessment within 1 week by the TCD and FL was noticed in table 7.

Table 7-P value comparing TCD with FL within 1 week

\begin{tabular}{|c|c|c|c|c|c|c|}
\hline \multirow{2}{*}{} & \multicolumn{2}{|c|}{ TCD } & \multicolumn{2}{c|}{ FL } & \multirow{2}{*}{ P value } \\
\cline { 3 - 7 } & Count & $\%$ & Count & \% & \multirow{2}{*}{ accuracy } \\
\cline { 2 - 7 } & Yes & 180 & $90 \%$ & 160 & $80.0 \%$ & 0.005 \\
\hline
\end{tabular}

$\mathrm{P}$ value was found to be 0.005 and showed that there was no significant difference between the correct assessment within 1 week by FL and by TCD.

Comparison between the percentage of correct assessment within 1 week by the FL and BPD was observed in table 8.

Table 8- P value comparing FL with BPD within 1 week

\begin{tabular}{|c|c|c|c|c|c|c|}
\hline & & \multicolumn{2}{|c|}{ BPD } & \multicolumn{2}{|c|}{ FL } & \multirow{2}{*}{$P$ value } \\
\hline & & Count & $\%$ & Count & $\%$ & \\
\hline \multirow{2}{*}{ accuracy } & yes & 120 & $60.0 \%$ & 160 & $80.0 \%$ & \multirow{2}{*}{$<0.001$} \\
\hline & no & 80 & $40.0 \%$ & 40 & $20.0 \%$ & \\
\hline
\end{tabular}

The $\mathrm{P}$ value was found to be $<0.001$ and showed that there was a highly significant difference between correct assessment within 1 week by FL and by BPD.

\section{Discussion:}

Many patients in Egypt due to socioeconomic reasons come for their first antenatal visit in third trimester. Most of them are uneducated come from remote areas. Also many being lactating mothers are unsure of their LMP or having irregular cycles. Because of non-availability of any dating scans or earlier ultrasound and uncertainty in LMP, it becomes very difficult to calculate their due dates, so many pregnancies considered to be preterm or posterm are wrongly classified. In the third trimester, various ultrasound parameters including BPD, which is one of the most commonly used parameters shows margin of error of 3-4 weeks from actual gestation. This is because of large biological variations in fetal skull shape and size. Management decisions become particularly difficult in conditions where there is growth restriction or growth acceleration and in planning induction for postdate pregnancy ${ }^{(3)}$. The femur length (FL) can be measured as early as 10 weeks gestational age because of its size and echogenicity. Correlation with true gestational age is within one week prior to 20 weeks gestational age, but falls to within 2.1 to 3.5 weeks in the third trimester. Including nonossified portions of the femur and not visualizing the full femur (femoral head/greater trochanter to femoral condyle) are the major sources of error in gestational age assessment by FL. The former over estimates and the latter 
underestimates gestational age (3). Transcerebellar diameter (TCD) represents an independent biometric parameter as shown in this study. The fetal cerebellum visualized as early as $10-20$ postmenstrual weeks. It grows in a linear pattern in the second trimester but the curve flattens in the third trimester (5). Cerebellum is not liable to change in form and size because of dense surrounding petrous ridges and occipital bone ${ }^{(5)}$. Due to this, TCD can be used where it is difficult to measure BPD or in cases where there are variations in size and shape of head. Normograms have been established for TCD and gestational age throughout pregnancy ${ }^{(6)}$. Naseem et al. (4) reported the better correlation of TCD with gestational age in the $2^{\text {nd }}$ and $3^{\text {rd }}$ trimester, its usefulness as growth assessing parameter in comparison with other routine ultrasound parameters. In our study, TCD was compared with FL and BPD in accuracy of assessment of gestational age in third trimester of pregnancy. We correlated between the three parameters, transcerebellar (TCD) and biparietal diameter (BPD) and femur length (FL), for accurate determination of gestational age in third trimester of pregnancy. FL and BPD were measured by using parameters of Hill et $\boldsymbol{a l} .{ }^{(7)}$. TCD was measured as widest diameter across both hemispheres and gestational age data for TCD was based on results of Chavez et al. ${ }^{(8)}$.

A total of 200 pregnant women in the third trimester pregnancy were assessed in this observational study in Department of Obstetrics and Gynecology, Damietta Hospital, Al-Azhar University (Outpatient) by two-dimensional ultrasound to assess an accurate method for assessment of gestational age in the third trimester of pregnancy. Examinations were performed after a verbal consent from the patient with the patient lying in the dorsal supine position. Two-dimensional ultrasounds were carried out. Fetal biometry and amniotic fluid volume were assessed. All women included in the study were subjected to history taking and clinical examination. We found that out of 200 patients, the TCD gave correct assessment of gestational age within 3 days in 118 patients $(59 \%)$ and within 1 week in 180 patients (90\%). While the FL gave correct assessment of gestational age within 3 days in 92 patients $(46 \%)$ and within 1 week in 160 patients $(80 \%)$. The least accurate was the BPD that gave correct assessment of gestational age within 3 days in 59 patients (29.5\%) and within
1 week in 120 patients (60\%). Rotmensch et al.

(9) measured the cerebellar diameter in cases of Down syndrome and found that cerebellar diameters in down syndrome fetuses were lesser than normal controls at all gestational age, by an average of $0.67-0.87 \mathrm{~mm}$. A ratio of 0.92 for observed/expected cerebellar diameter gave a sensitivity of $21 \%$, specificity of $95 \%$ and PPV $1.66 \%$ and $0.50 \%$ in a population with risk of having Down syndrome of 1 in 250 and 1 in 750 respectively. However, this difference in cerebellar size was too small to be used clinically. In a study done by Chavez et al. ${ }^{(10)}$ the concordance between the actual and predicted gestational age by TCD was high. The agreement was superior in the second trimester $(\mathrm{r}=0.93)$ compared to the third trimester. Chavez et al. (11) studied TCD in twin pregnancies and concluded that the agreement between the actual gestational age and the predicted gestational age by TCD was comparable to that of singleton pregnancy. In another study by Chavez et al. (8) the concordance between the actual gestational age and the predicted gestational age by TCD was high for both IUGR and large fetus. Joshi ${ }^{(12)}$ measured TCD in 594 singleton pregnancies in Nepalese population. They found that the gestational age and TCD (50th percentile in $\mathrm{mm}$ ) coincided well till 20th week of gestation. They observed no significant clinical difference between the nomogram created by them and the previously published nomogram in gestational age between 21st and 28th weeks. But, they observed significant differences between their nomogram and the previously published nomograms in third trimester.Goel et al. ${ }^{(13)}$ evaluated the fetal cerebellar development ultrasonographically which in turn is helpful in assessing the fetal gestational age. They showed that TCD was well correlated with gestational age. So, transverse cerebellar diameter was a good marker for gestational age estimation and can be used in cases that were not sure about their LMP. They offered the normal range of the cerebellar measurements throughout gestationa. These values may allow intrauterine assessment of the development of the cerebellum as well as the posterior fossa. Naseem et al. ${ }^{(14)}$ investigated the posterior cranial fossa of the fetus and confirmed the capability of the ultrasound to demonstrate the anatomy of the fetal posterior cranial fossa. The vermis and cisterna magna as well as the cerebellar hemispheres could be demonstrated 
easily. They also proposed a systematic approach to prenatal ultrasound examination of the posterior fossa. They suggested that the use of fetal transcerebellar diameter in utero between 17 and 40 weeks of gestation is a useful indicator of accurate gestational age. In the present work TCD showed a useful indicator of accurate gestational age in third trimester of pregnancy. Naseem et al. (14) performed a study on 228 patients comparing TCD and BPD accuracy in the third trimester. They found that there was a good correlation between the multiple growth parameters and TCD and concluded that TCD is more reliable method of gestational age determination in third trimester than BPD. Prssad and Likhitha ${ }^{(15)}$ studied an equation that correlates between the GA and the TCD and detected a good correlation between the GA and TCD throughout the third trimester and even in the case of intra uterine growth retardation (IUGR).Another study done by Naseem $\boldsymbol{e t}$ al. (4) on 327 patients pregnant in their third trimester comparing TCD with FL showed that TCD was more reliable method of gestational age determination in third trimester than FL.Akl et al. ${ }^{(16)}$ performed a study in Egypt on 150 pregnant women in their third trimester to determine the accuracy of the TCD in assessment of gestational age and he concluded that the TCD is a reliable method for assessment of gestational age in third trimester of pregnancy.

Prssad and Likhitha ${ }^{(15)}$ studied an equation that correlates between the GA and the TCD and detected a good correlation between the GA and TCD throughout the third trimester and even in the case of intra uterine growth restriction, that equation was as follow: $\mathrm{GA}=-$ $0.007(\mathrm{TCD})^{2}+1.1032(\mathrm{TCD})+0.2463$. Reddy $\boldsymbol{e t}$ al. ${ }^{(17)}$ evaluated accuracy of predicting GA used Foetal Transcerebellar Diameter (TCD) and compared between TCD and other existing parameters in evaluating GA in 15 to 40 weeks of gestation. They showed that TCD was an accurate parameter in estimation of gestational age in second and third trimesters as its values are in close relation with that of GA by LMP. It is also better predictor of the gestational age when compared to other parameters especially in third trimester.

Conclusion:

From the study, we concluded that TCD was the most accurate method for assessment of gestational age in third trimester followed by FL, and the least accurate is the BPD. Also by combining accuracy of TCD $(90 \%)$ and that of FL (80\%) we can be near certain of gestational age in most of our patients even if they are unsure of their dates.

Recommendations:

1. Measuring the TCD as routine in the third trimester as it has the same accuracy as the current fetal biometry (BPD and FL).

2. Further study assessing the accuracy of the TCD in estimation of the gestational age in extremes of fetal growth; IUGR and fetal macrosomia.

3. We recommend toconduct this study on larger sample size for further documentation of the proposed assumption.

\section{References:}

1. Skupski DW, Owen J, Kim S et al. (2016): The NICHD fetal growth studies: development of a contemporary formula for estimating gestational age from ultrasound fetal biometrics. AJOG., 214 (1):74-82.

2. Leibovitz Z, Shiran C, Haratz $\mathrm{K}$ et al. (2016): Application of a novel prenatal vertical cranial biometric measurement can improve accuracy of microcephaly diagnosis in utero. Ultrasound Obstet. Gynecol., 47(5):593-602.

3. Lemer JP (2004): Fetal growth and well being. Obstet. Gynecol. Clin. North. Am., 31: 159- 176.

4. Naseem F, Ali S, Basit U et al. (2014): Assessment of gestational age: Comparison between transcerebellar diameter versus femur length on ultrasound in third trimester of pregnancy. Professional Med. J., 21(2): 412-417.

5. Kuklisova M, Aljabar P, Srinivasan $L$ et al. (2011): A dynamic 4D probabilistic atlas of the developing brain. Neuroimage, 54: 2750-2763.

6. Baschat AA. (2011): Fetal growth disorders. In: High Risk Pregnancy. Baschat AA, editor.. St. Louis. Saunder. london,pp: 173-179.

7. Hill LM, Guzick D, Hixson J et al. (1992): Composite assessment of gestational age: a comparison of institutionally derived and published 
regression equations. Am. J. Obstet. Gynecol.,166:551-556.

8. Chavez MR, Ananth CV, Smulian JC et al. (2007): Fetal transcerebellar diameter measurement for prediction of gestational age at the extremes of fetal growth. Journal of Ultrasound in Medicine, 26: 1167-1171.

9. Rotmensch S, Goldstein J, Liberati M et al. (1997): Foetal transcerbellar diameter in Down's syndrome. Obstet. Gynecol., 89(4): 534- 537.

10. Chavez MR, Ananth CV, Smulian JC et al. (2004): Foetal transcerebellar diameter measurement with particular emphasis in the third trimester: a reliable predictor of gestational age. Am. J. Obstet. Gynecol., 19: 1979-1984.

11. Chavez MR, Ananth CV, Kaminsky LM et al. (2006): Foetal transcerebellar diameter measurement for prediction of gestational age in twins. American Journal of Obstetrics and Gynecology, 195(6): 1596-1600.

12. Joshi BR (2010): Foetal transcerebellar diameter nomogram in Nepalese population. Journal of Institute of Medicine, 32(1): 19-23.
13. Goel $P$, Singla $M$, Ghai $R$ et al. (2010): Transverse cerebellar diameter-a marker for estimation of gestational age. J. Anat. Soc. India, 59(2): 158-161.

14. Naseem $\mathrm{F}$, Fatima $\mathrm{N}$, Yasmeen $\mathrm{S}$ et al. (2013): Comparison between transcerebellar diameters with biparietal diameter of ultrasound for gestational age measurement in the third trimester of pregnancy. J. Coll. Physicians Surg. Pak., 23(5): 322-325.

15. Prssad BS and Likhitha S (2014): Cerebellar measurements with ultrasonography in evaluation of fetal age. Journal of Dental and Medical Sciences , 13(9): 49-56.

16. Akl S, Mohammed M, Bahaa El-din A et al. (2014): Accuracy of transcerebellar diameter at the third trimester in estimating the gestational age in singleton pregnancy. Med. J. Cairo Univ., 82(1): 879-884.

17. Reddy RH, Prashanth $K$ and Ajit $M$ (2017): Significance of foetal transcerebellar diameter in foetal biometry: a pilot study. Journal of Clinical and Diagnostic Research, 11(6):1-4. 